\title{
ALFABETO VISUAL Y CALIGRAFÍA COMO HERRAMIENTA TERAPÉUTICA CON PERSONAS CON DEMENCIA TEMPRANA
}

\author{
Lorena López Méndez. \\ lorena.Iopez.mendez@unir.net
}

Fecha de Recepción: 1 Febrero 2018

Fecha de Admisión: 10 Abril 2018

\section{RESUMEN}

El Proyecto "Alfabeto Visual" Arte y Salud Alzhéimer, es un proyecto que pretende acercar la educación artística contemporánea a personas con demencia temprana a través de la caligrafía y los refranes, a sus familiares- cuidadores y personal sanitario. Permitiéndoles participar en una serie de actividades lúdicas, creativas y culturales. El taller forma parte del programa de educación artística diseñado e implementado durante el 2014 y 2015 en el Centro de Referencia Estatal de atención a personas con enfermedad de Alzhéimer y otras Demencias de Salamanca, dependiente del Instituto de Mayores y Servicios Sociales (IMSERSO).

Los objetivos del programa están enfocados no sólo en mejorar la calidad de vida y preservar las capacidades cognitivas a través de la utilización del Arte como herramienta, sino también en sensibilizar a la sociedad, erradicando estigmas asociados a la enfermedad en fases incipientes, donde todavía el participante puede participar y comunicarse.

Los resultados del programa nos permiten considerar que el Arte puede ofrecer una nueva oportunidad de aprendizaje y un soporte de ayuda terapéutica tanto individual como cooperativo, desarrollar habilidades cognitivas y funcionales, ampliar recursos emocionales y disfrutar de la experiencia, fortaleciéndose la seguridad y autoestima en sí mismos, ante la capacidad de formar parte activa del programa.

Palabras clave: educación artística; caligrafía; refranes; demencia temprana; alzhéimer

\section{ABSTRACT}

Visual alphabet and calligraphy as a therapeutic tool with people with early dementia.

The "Visual Alphabet "Project: Art and Health Alzheimer's, is a project that aims to bring contemporary art education, proverbs and caligraphy for people with early dementia, their family members, caregivers and healthcare personal. Allowing them to participate in funny, creative and cultural activities. This article exposed arts education program designed and implemented during 2014 and 2015 in the State Reference Center of care for people with Alzheimer's disease and other demen- 


\section{ALFABETO VISUAL Y CALIGRAFÍA COMO HERRAMIENTA TERAPÉUTICA CON PERSONAS CON DEMENCIA TEMPRANA}

tias of Salamanca, under the Aging and Services Sociale Institute (IMSERSO). The program objectives are focused not only on improving the quality of life and preserve cognitive abilities through the use of art as a tool, it also raise awareness eradicating stigma associated with the disease in early stages, where the participant can participate and communicate. The program results allow us to consider that arts education can offer a new opportunity for individual and collaborative learning, develop cognitive and functional abilities, expand emotional resources and enjoy the experience, strengthen security and self-esteem in themselves, with the ability to be an active part of the program.

Keywords: art education; calligraphy; proverbs; early dementia; Alzheimer

\section{INTRODUCCIÓN}

Los pacientes con enfermedad de Alzhéimer (EA) suelen experimentar síntomas neuropsiquiátricos que disminuyen su calidad de vida y bienestar. En ausencia de una perspectiva de futuro en la cura de esta enfermedad a corto plazo, se precisan tratamientos e intervenciones con carácter global, es decir tratamientos tanto farmacológicos como no farmacológicos. En intervenciones no farmacológicas (TFN) o terapéuticas es donde el arte y por ende la Educación artística juega un papel esencial para la transformación y acción social en este contexto, porque los participantes con esta enfermedad pueden apreciar y producir arte visual, mediante un programa bien estructurado y apropiado a las necesidades de cada participante.

Por estos motivos germinó el diseño e implementación de un programa de Educación artística para este perfil de participantes, gracias al amparo de la Beca Predoctoral PIRTU (ORDEN EDU/1204/2010, de 26 de Agosto), financiada por la Junta de Castilla y León y el Fondo Social Europeo.

El taller que se describe a continuación forma parte de un programa más amplio de una veintena de talleres. En esta ocasión se pretende emplear la caligrafía y la escritura como herramienta artística. Asimismo, se empleó como pretexto la caligrafía y letraser, pues la mayoría de los participantes aprendieron a escribir con pluma y su tipo de escritura es de carácter caligráfico como se puede analizar en su escritura. Se comenzó a trabajar empleando como base una serie de poemas, palabras y frases en las que la tipografía o bien caligrafía configuran una especie de imagen visual o Poesía visual que les permitiese expresarse visualmente lo que las palabras y conjunto de palabras que quieren transmitir. Para tal fin, nos servimos de dichos populares conocidos por los participantes como es el refranero español.

\subsection{Objetivos generales del Proyecto.}

Los objetivos planteados, responden a dos niveles de intervención, tanto psicosocial como artístico:

Mejorar la calidad de vida, autoestima y sistemas de cuidado de personas con Alzhéimer u otras demencias y sus cuidadores familiares.

Favorecer conexiones con su contenido vital pasado y presente a través del Arte y la experiencia estética.

Fomentar la participación y comunicación del usuario con la Producción Cultural.

Preservar las capacidades cognitivas de los participantes el mayor tiempo posible.

Sensibilizar a la sociedad erradicando estigmas asociados a la enfermedad.

\subsection{Objetivos específicos del taller "Alfabeto Visual"-Poema Visual con Caligramas. Objetivos artísticos \\ Conocer el concepto Caligrama. \\ Narrar un poema o refrán español que conozca el participante.}


Transmitir un mensaje mezclando texto e imagen-poema visual.

Crear una composición mediante la utilización de diferentes estilos tipográficos.

\section{Objetivo terapéutico}

Recuperar la escritura como medio para fomentar la psicomotricidad fina.

Establecer comunicación y diálogo entre los participantes, poniendo en cuestión temáticas de interés.

Emplear el refranero español como recurso para fomentar la memoria y el recuerdo.

\section{METODOLOGÍA.}

\subsection{Contexto de actuación.}

El programa se implementó en colaboración con el Centro de Referencia Estatal de Atención a Personas con Enfermedad de Alzheimer y otras Demencias de Salamanca (CRE) (http://www.crealzheimer.es/crealzheimer_01/index.htm). Este Centro situado en Salamanca, se configura como un centro sociosanitario especializado y avanzado en investigación, análisis, conocimiento, evaluación y formación sobre el Alzheimer y otras demencias y en atención e intervención con las personas afectadas y sus familias.

\subsection{Participantes.}

En el programa se consideraron elegibles todos los usuarios del Centro (CRE) en fase moderada,(GDS 4 a 5 en la escala de Reisberg) y en MMSE presentaban una puntuación que iba desde 12 a $27(M=20, S D=3,6)$. A todos ellos $(N=17)$ se les ofreció la posibilidad de incorporarse al programa. Finalmente participaron de manera continuada 16. 8 usuarios (7 mujeres y 1 hombre) del centro de día y 8 usuarios (7 mujeres y 1 hombre) de unidades de convivencia, seleccionadas por su interés por el Arte y sus procesos técnicos.

\section{MÉTODO.}

El programa de educación artística se configuro en 28 sesiones, y el taller descrito se llevó a cabo durante 6 sesiones, durante 5 meses a razón de una sesión semanal de 1 hora y 30 minutos en formato grupal (2 grupos de 8 usuarios máximo). La intervención se realizó entre las 15:00 y las 19:00 p.m. en un espacio designado específicamente para la actividad y aislado de las unidades de convivencia y el centro de día. Las sesiones se llevaron a cabo con la presencia de una experta en Educación artística, una educadora social y una auxiliar de clínica.

La actividad "Alfabeto Visual", se desarrolló en tres fases. En primer lugar, fase de apreciación, se comienza con el visionado de imágenes artísticas en las que los participantes podían ver una serie de caligramas de diferentes artistas que emplean la técnica del caligrama como herramienta para sus proyectos artísticos. (Veáse, Tabla 1). Está técnica consiste en frases o poemas en los que la tipografía, caligrafía o el texto crea una especie de imagen visual (poesía visual) que expresa visualmente lo que la palabra o palabras dicen. (Véase, Fig. 1). 


\section{ALFABETO VISUAL Y CALIGRAFÍA COMO HERRAMIENTA TERAPÉUTICA CON PERSONAS CON DEMENCIA TEMPRANA}

Tabla 1. Obras representativas que configuran la presentación.

\begin{tabular}{|c|c|c|}
\hline ARTISTA & OBRA/TÉCNICA & FUENTE \\
\hline $\begin{array}{l}\text { Guillaume } \\
\text { Apollinaire, }\end{array}$ & $\begin{array}{l}\text { La paloma } \\
\text { apuñalada y el } \\
\text { surtidor. } \\
\text { Simmias de } \\
\text { Rodas, siglo IV } \\
\text { A.C. } \\
\text { Hombre con } \\
\text { sombrero.1880. } \\
\text { Caballo.1880. }\end{array}$ & $\begin{array}{l}\text { http://empanadediseno.wordpress.com/tag/dadaismo- } \\
\text { constructi- vismo-caligramas-leger-apollinaire-mallarme- } \\
\underline{\text { huidobro-lizzitsky- matta/ }} \\
\text { http://lenguayliteratura10.wordpress.com/tag/apollinaire }\end{array}$ \\
\hline León Ferrari. & $\begin{array}{l}\text { 'P4CR' } 1979 . \\
\text { Tinta y Letraset } \\
\text { sobre papel. } 32,8 \\
\text { x 21,5 cm. } \\
\text { Sin título. } 1979 . \\
\text { Letter to a } \\
\text { general. } 1963 .\end{array}$ & $\begin{array}{l}\text { Fundación Augusto y León Ferrari. Archivo y Colección } \\
\text { (Buenos Aires). } \\
\text { http://www.museoreinasofia.es/exposiciones/leon- } \\
\text { ferrari-mira-schendel-alfabeto-enfurecido }\end{array}$ \\
\hline Shirin Neshat. & $\begin{array}{l}\text { Roja, } 2012 . \\
\text { Impresión } \\
\text { digital.153 x } \\
114.9 \mathrm{~cm} .\end{array}$ & $\begin{array}{l}\text { http://papeldeperiodico.com/2014/03/el-arte-traves-de- } \\
\text { las-mujeres-shirin-neshat-1957-actualidad/ }\end{array}$ \\
\hline $\begin{array}{l}\text { Mira } \\
\text { Schendel. }\end{array}$ & $\begin{array}{l}\text { Sin título, de la } \\
\text { serie Objetos } \\
\text { Gráficos, } 1973 \text {. } \\
\text { Letraset sobre } \\
\text { papel de arroz } \\
\text { entre láminas de } \\
\text { acrílico transpa- } \\
\text { rentes. 55,5 cm x } \\
55,5 \mathrm{~cm}\end{array}$ & $\begin{array}{l}\text { http://www.museoreinasofia.es/exposiciones/leon- } \\
\text { ferrari-mira-schendel-alfabeto-enfurecido }\end{array}$ \\
\hline
\end{tabular}


Fig.1. Representación del Caligrama.Colocamos la letra del abecedario de la transferencia (letraset) que nos interese sobre el dibujo, de manera que se construye el caligrama. Fotografía: Lorena López.

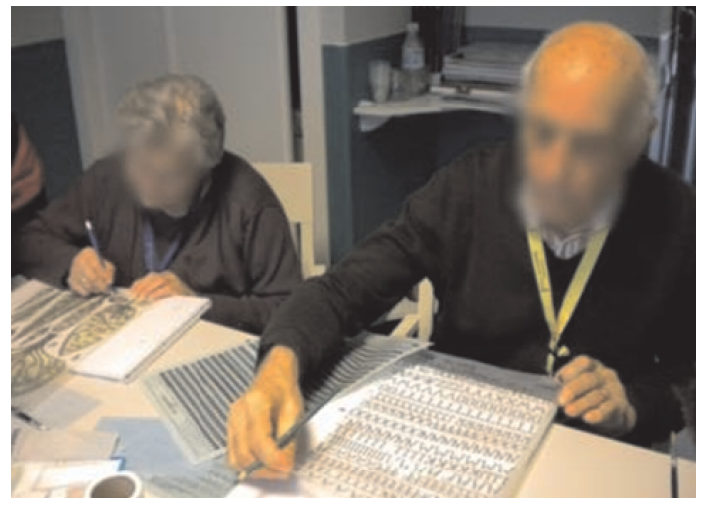

Posteriormente del visionado de la presentación, iniciamos la fase debate, a partir de una serie de preguntas motor, que son las siguientes: ¿Se puede dibujar con letras? y ¿Se podría dibujar el Refranero Español?. A continuación, los participantes realizaron su propia serie de caligramas entorno al refranero español, donde se reflexiona sobre la veracidad de los dichos populares. Y la propuesta se planteó partiendo del dicho popular "Nunca es tarde si la dicha es buena".(Vease, Fig. 2 y $3)$.

Fig.2. Participante T. El arbol de la esperanza. Caligrama y tempera. 26,5 x $27 \mathrm{~cm}$. Enero 2011. Fotografía: Lorena López.

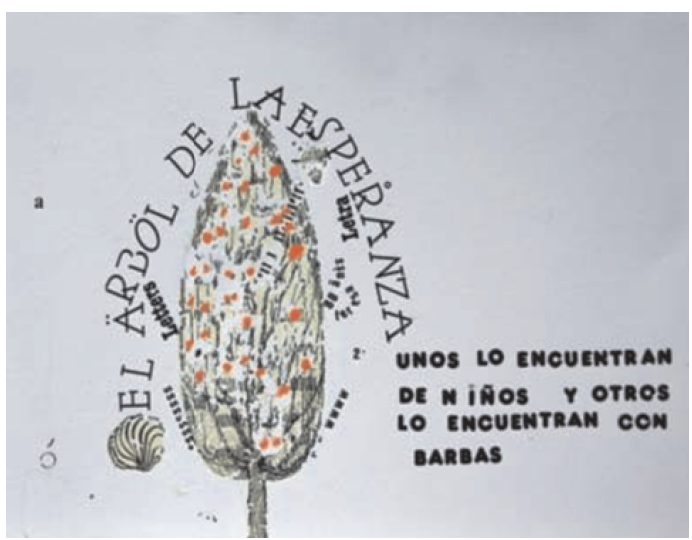




\section{ALFABETO VISUAL Y CALIGRAFÍA COMO HERRAMIENTA TERAPÉUTICA CON PERSONAS CON DEMENCIA TEMPRANA}

Fig.3.Participante C. Ande yo caliente, riase la gente. Caligrama y tempera. $26,5 \times 27 \mathrm{~cm}$. Enero 2011.Fotografía: Lorena López.

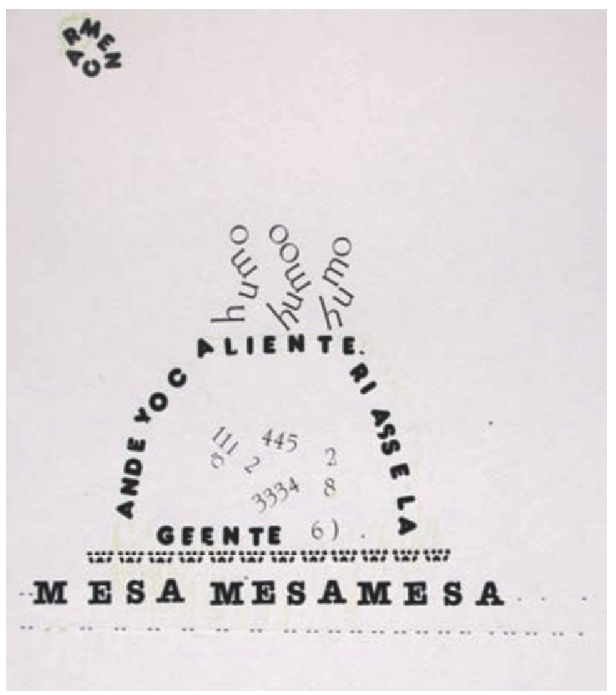

Seguidamente, se propone a los participantes que pusiesen en práctica y empleasen la caligrafía como una manera de expresión de un tema o refrán que para ellos sea cercano, para terminar realizando la representación del dibujo sobre el que iría el texto.

Por último, fase producción, representaron el caligrama, transfirieron las letras sobre la representación e iluminaron el caligrama si lo consideraban oportuno.

La metodología implementada en el taller fue de carácter cualitativo, concretamente se llevó a cabo una rigurosa observación participante. Así como entrevistas no estructuradas a auxiliares-cuidadoras y personal socio-sanitario. Uno de los objetivos era obtener perspectiva de terceros sobre cómo estaba diseñada la intervención. Todos los datos fueron registrados como notas de campo 0 capturados con una cámara digital fotográfica- grabadora y transcritas posteriormente. Respecto al análisis de datos fueron recogidos mediante el programa de análisis de datos atlas. ti (versión 7).

\section{RESULTADOS.}

El análisis de resultados y en concreto su evaluación, se llevó a cabo de manera consensuada respondiendo a un formulario resumen (Ullán et al. 2012) acerca de cada participante, entre las educadoras que formaban el equipo. El nivel de desacuerdo solía ser bajo y en caso de duda siempre se seleccionaba la opción de respuesta que menos compromiso implicaba.

En relación a la pregunta 4.¿Ha manifestado interés y atención en el proceso del taller?.De las 104 evaluaciones recogidas, hemos obtenido un 65\% $(n=68)$ en la respuesta a. Sí de forma continua durante toda la duración del mismo. Un $21 \%(n=22)$ en la respuesta b. Sí pero con intervalos. Un $14 \%(n=15)$ en la c.No, y por último en la d. No se podría decir, un $0 \%(n=0)$.

Ante estos resultados, consideramos la participación, el esfuerzo y el interés mostrado por los participantes, factores claves en nuestro modelo educativo, siendo este de garantía pues presentamos una valoración alta $86 \%$ en su interés y atención durante el proceso del taller. Por el contrario 
el porcentaje de participantes que consideramos que no han manifestado interés y atención es irrelevante dentro del total de participantes analizados, estando este valor entorno al 14\%. También destacamos un porcentaje nulo de participantes de los que no se podría precisar su interés y atención en el proceso del taller.

En general la asistencia y participación en el taller ha sido muy positiva del $90 \%(n-7$ de 10), pues en la mayoría de los casos todos querían asistir al taller, aunque en alguna ocasión sea como mero espectador y salvo en dos ocasiones dos participantes abandonaron el aula -taller, uno por desavenencias con el grupo y otro por estado de ansiedad y agitación previo al taller.

Respecto a la cuestión 5.¿Ha completado el trabajo del taller?. Hemos alcanzado en la respuesta a. Sí, un $47 \%(n=49)$. En la b. A medias, un 50\%(n=52) y en la c. No, un 3\%(n=3). Si analizamos estas respuestas podemos afirmar que los participantes precisan más tiempo del estipulado para realizar la obra.

Por último, contestando a la pregunta 6.¿Ha disfrutado del taller?. Se ha obtenido los siguientes resultados, en la respuesta a. Sí claramente, lo afirma o se observa con ambigüedad, un $40 \%$ $(n=42)$. En la respuesta b. Sí, posiblemente(es una deducción de los técnicos) un 37\%(n=39). En la c. No podemos afirmar nada al respecto, un $22 \%(n=23)$ y por último d. No se podría decir, un $1 \%(n=1)$.

En este punto tenemos que señalar que un $77 \%$, ha disfrutado en el taller, el ambiente generado en el mismo ha sido agradable y distendido. Frente a un $23 \%$ que no podríamos especificar.

A raíz de la implementación de este proyecto por diversas instituciones desde hace cuatro años, consideramos conveniente continuar diseñando estudios e investigando sobre esta problemática, pues las técnicas de participación creativa y artística en el cuidado de la demencia, nos permiten trabajar hacia la respuesta a la pregunta de qué mecanismos están en juego en la evidencia experimental de los resultados positivos que observamos en el campo tanto del contexto de las Demencias como en el de la Educación artística en contextos no formales.

\section{CONCLUSIONES.}

La actividad descrita funcionó positivamente, una vez que fue repetida en varias ocasiones, para reforzar su proceso de enseñanza aprendizaje. Este detalle se viene observando durante la implementación de la programación en completo. Es preciso repetir cada actividad- taller para que los usuarios poco a poco vayan asimilando el proceso o incluso la temática, aunque esta es de fácil asimilación, pues siempre tienen relación con temas de interés para los y las participantes, ya que están intrínsecamente en su contenido vital.

El debate generado durante el taller, acabo teniendo relación directa con el refranero, proverbios y dichos españoles. Los usuarios del taller son conocedores de estos dichos populares provenientes de experiencias vividas. Incluso terminaron realizando un "concurso" para ver quién era el que más refranes conocía.

En la fase de producción del taller pudimos observar primero que el empleo del Refranero como "detonante", es un tema que los usurarios conocen bien y pueden enseñarnos a nosotros más que nosotros a ellos, produciéndose generar un cambio de roles y segundo, el hecho de hacer uso de letraset, facilita el poder escribir a personas cuya psicomotricidad fina es reducida. Asimismo las letraset, nos ofrecen un factor sorpresa, ya que simplemente con presionar levemente con un lápiz sobre las letras, conseguimos letras perfectas que aumentan el interés de los participante para continuar probando.

De esta manera conseguimos fomentar su autoestima, gracias al hecho de que los participantes de manera natural transmitan sus conocimientos a los educadores, de tal manera que se fomen- 


\section{ALFABETO VISUAL Y CALIGRAFÍA COMO HERRAMIENTA TERAPÉUTICA CON PERSONAS CON DEMENCIA TEMPRANA}

te un cambio de roles. Es decir el educador se convierte en participante y el participante en educador. De esta manera dejaron manifesta su sabiduría como es el caso de la participante T, con su refrán: "El árbol de la es- peranza unos lo conocen de niños y otros cuando tienen barbas" o la participante C. "Ande yo caliente ríase la gente".

Otros participantes reconocieron que el proceso de aprender nuevos conceptos artísticos así como el diálogo entre compañeros o entre educador-usuario les ayudó a permanecer cognitivamente activos. Como afirmaron varios participantes: "Me gustan mucho los refranes me hacen pensar". [...] "Me gusta estas letras, así puedo escribir" "No sabía que se puede hacer Arte con letras, cada día se puede seguir descubriendo" [...] "Lo mejor de este taller es el buen rato que pasamos, si te equivocas no importa [...]. " ¡Qué divertido, podemos seguir viendo cosas nuevas, una oportunidad para ver!' [...].

Los beneficios psicosociales percibidos en los datos cualitativos recogidos durante la participación en el taller fueron: mayor estimulación cognitiva, sentido de propósito y sentido de utilidad, reminiscencia, alegría por aprender cosas nuevas, contacto con otros participantes, aceptación de capacidades, no temor al error. Estos beneficios contribuyen a una mayor transferencia y transformación de la investigación en Educación artística.

Respecto a las opiniones vertidas por el personal sociosanitario y los familiares-cuidadores que cuidan a los personas con EA, la cuestión de que es lo que la persona con demencia todavía puede hacer es una constante (Chancellor, Duncan \& Chatterjee, 2014) y este taller lo perciben como un tratamiento capaz de aminorar síntomas neuropsquiatrico, mejorando su calidad de vida y bienestar, combinándolo con otras terapias.

A día de hoy en ausencia de perspectivas a corto plazo de la existencia de cura para esta enfermedad degenerativa, precisamos de un tratamiento global con la unión de intervenciones farmacológicas y no farmacológicas. Por lo tanto el arte y sus diferentes herramientas, técnicas y conceptos, pueden permitirnos actuar en una misma dirección, trabajando diversas habilidades y déficits de la persona, como también adaptar las condiciones del participante, a su situación y habilidades. Por lo tanto son necesarios más programas y talleres de artes para personas con EA como el descrito, pues proporciona bienestar y mejora en los síntomas neuropsiquiatricos, la autoestima y el comportamiento social de quien la práctica.

\section{CONTRIBUCIONES.}

Una vez analizados los resultados podemos señalar una serie de beneficios psicosociales de bienestar físico, mental y emocional que han contribuido a una mejor calidad de vida y bienestar de los participantes por medio de un aumento de la actividad y por consiguiente del capital social, la fuerza y capacidad cognitiva, la creación de relaciones significativas y el aumento de auto- estima. En general ha contribuido a un mejor funcionamiento psicológico del participante.

Los participantes con demencia pueden transmitir la profundidad, la proporción y el detalle en su obras de arte, sin embargo la mayoría de los pacientes con demencia moderada no son capaces de crear formas y figuras que representen una idea que está en sus memoria, pero pueden todavía producir obra utilizando el color y la composición de manera abstracta, creando imágenes geométricas. (Stewart, 2004).

\section{BIBLIOGRAFÍA.}

Chancellor, B. Duncan, A \& Chatterjee, A. (2014). Review Art Therapy for Alzheimer's Disease and Other Dementias. Journal of Alzheimer's Disease 39 (2014) 1-11.doi 10.3233/JAD-131295

Geue K, Goetze H, Buttstaedt M, Kleinert E, Richter D, Singer, S. (2010). An overview of art therapy interventions for cáncer patients and the results of research. Complement Ther Med 18, 160170. 
Gray AE. (2011). Expressive arts therapies:Working with survivors of torture. Torture 21, 39-47.

López, L. (2014). Educación artística en personas con Demencia Temprana. International Journal of Developmental and Educational Psychology. INFAD Revista de Psicología, №1-Vol.1, ISSN: 0214-9877. 527-534.

Miller BL, Hou CE. (2004). Portraits of artists: Emergence of visual creativity in dementia. Arch Neurol 61, 842-844.

Stewart, EG. (2004). Art therapy and neuroscience blend:Working with patients who have dementia. Art Ther J Am Art Ther Assoc 21, 148-155.

Stuckey HL, Nobel J. (2010). The connection between art, healing, and public health: A review of current literature. Am J Public Health 100, 254-263.

Ullán, A.M. Belver,M.H. Badia, M. Moreno,C. Garrido, E. Gómez-Isla, J. Gonzaléz-Ingelmo,E. Delgado , J. Serrano, I. Herrero, C. Manzanera,P \& Tejedor, L. (2012). Contributions of an artistic educational program for older people with early dementia: An exploratory qualitative study. Dementia. 0(0). doi: 10.1177/1471301211430650. 1-22. 
